The Astronomical Journal, 115:909-914, 1998 March

(C) 1998. The American Astronomical Society. All rights reserved. Printed in U.S.A.

\title{
CHEMICAL ABUNDANCE CALIBRATIONS FOR THE NARROW-LINE REGION OF ACTIVE GALAXIES
}

Thaisa Storchi-BergmanN and Henrique R. Schmitt

Departamento de Astronomia, Instituto de Física, Universidade Federal do Rio Grande do Sul, C.P. 15051, CEP 91501-970, Porto Alegre, RS, Brazil

AND

Daniela Calzetti and AnNe L. Kinney

Space Telescope Science Institute, 3700 San Martin Drive, Baltimore, MD 21218

Received 1997 August 14; revised 1997 November 14

\begin{abstract}
We investigate two chemical abundance calibrations for the narrow-line region (NLR) of active galaxies in terms of three easily observable optical emission-line ratios, namely, [O $\mathrm{III}] \lambda \lambda 4959,5007 / \mathrm{H} \beta$; [N II] $\lambda \lambda 6548,6584 / \mathrm{H} \alpha$; and [O II $] \lambda 3727 /[\mathrm{O}$ III] $\lambda \lambda 4959,5007$. The calibrations are obtained from a grid of models on the assumption that the main process responsible for the production of these lines is photoionization by a "typical" active galactic nucleus continuum. The elemental abundances vary with oxygen, except nitrogen, which is assumed to be a product of secondary nucleosynthesis. The calibrations are calculated for the range $8.4 \leq 12+\log (\mathrm{O} / \mathrm{H}) \leq 9.4$ and tested using NLR data for a sample of Seyfert galaxies and LINERs that have $\mathrm{H}$ II regions in the vicinity of the nucleus. The gaseous abundances of these $\mathrm{H}$ II regions have been determined in previous works, and the NLR abundances are obtained on the assumption that they can be extrapolated from those of the $\mathrm{H}$ II regions. The calibrations work very well for the Seyfert galaxies, yielding abundance values that agree with those obtained from the $\mathrm{H}$ II regions, and can thus be used for quick estimates of the chemical abundances of the NLRs. For the LINERs, the calibrations yield lower values than those derived from the corresponding $\mathrm{H}$ II regions, suggesting that the assumptions of the models do not apply for them and that there are different physical processes at work in the NLR of the LINERs.
\end{abstract}

Key words: galaxies: active — galaxies: ISM — galaxies: nuclei — galaxies: Seyfert

\section{INTRODUCTION}

Since the works of Ferland \& Netzer (1983) and Halpern $\&$ Steiner (1983) on the modeling of the narrow-line region (NLR) of active galactic nuclei (AGNs), which were followed by many subsequent works (e.g., Stasińska 1984; Ferland \& Osterbrock 1986; Binette, Robinson, \& Courvoisier 1988), it has been known that the relative fluxes of the stronger emission lines can be successfully reproduced under the assumption that the main excitation mechanism of the NLR is photoionization. Alternative photoionization models include varying proportions of matter-bounded and ionization-bounded clouds in the NLR (Viegas-Aldrovandi 1988; Binette, Wilson, \& Storchi-Bergmann 1996), which are better suited to reproduce both the high- and the lowexcitation emission-line ratios. Viegas-Aldrovandi \& Contini (1989) have also investigated the effect of shocks, concluding that clouds with shock velocities below $300 \mathrm{~km}$ $\mathrm{s}^{-1}$ are radiation dominated if the ionization parameter is greater than $U=10^{-4}$. Viegas-Aldrovandi \& Gruenwald (1990) have also shown that, besides shocks, relativistic particles have to be taken into account in order to reproduce the spectra of LINERs. More recent studies of the effect of shocks in the NLR or ENLR (extended narrow-line region, an expression sometimes used to indicate that the NLR is obviously extended, usually to kpc scales) include the works of Viegas \& de Gouveia Dal Pino (1992), Sutherland, Bicknell, \& Dopita (1993), and Dopita \& Sutherland (1995).

The conical shape observed for the ENLR in a number of Seyfert galaxies seems to favor photoionization by a nuclear source as the ionizing mechanism (Wilson 1996 and references therein). This is confirmed by long-slit spectroscopy along the extended gas for a few Seyfert galaxies, such as NGC 3281 (Storchi-Bergmann, Wilson, \& Baldwin 1992),
Mrk 573 (Tsvetanov \& Walsh 1992), and NGC 5643 (Schmitt, Storchi-Bergmann, \& Baldwin 1994). The variation of the emission-line ratios with distance from the nucleus in these three galaxies can be reproduced by a sequence of models with varying ionization parameters, even when there is evidence of the presence of shocks with velocities $\sim 150 \mathrm{~km} \mathrm{~s}^{-1}$ in the gas (e.g., NGC 3281).

Most works devoted to reproducing the emission-line ratios of the NLR or ENLR via photoionization models have adopted a solar composition for the gas. Collecting NLR emission-line ratios from a sample of 180 LINERs and Seyfert 2 galaxies, Storchi-Bergmann \& Pastoriza (1990), showed that the data are better reproduced if a range of nitrogen abundances, from solar to 4 times solar, is adopted.

In order to verify this result, Schmitt et al. (1994), StorchiBergmann, Wilson, \& Baldwin (1996b), and StorchiBergmann et al. (1996a) obtained long-slit spectroscopy of a sample of AGNs with $\mathrm{H}$ II regions in the vicinity of the nucleus, such that the abundances could be determined from the spectra of the $\mathrm{H}$ II regions rather than by using models for the NLR (on the assumption that the nuclear, or NLR, abundance is the same as that of the $\mathrm{H}$ II regions, because of their proximity to the nucleus). The derived nuclear oxygen abundances range from solar to 2-3 times solar, and the nitrogen shows a secondary behavior, such that its abundance can reach up to 4-5 times solar, in accordance with the previous results from the modeling of the NLR.

Nevertheless, the number of active galaxies for which such determinations are available is small, and there are also several active galaxies that lack $\mathrm{H}$ in regions close to the nuclei, so that the above method cannot be used. In this 
work, we use the $\mathrm{H}$ II region abundances derived previously to infer that of the NLR and constrain, via a grid of photoionization models, the average physical parameters of the NLR. We then investigate the relation between the emission-line ratios and chemical abundances. Under a few assumptions, discussed below, we obtain two calibrations that allow the derivation of the chemical abundance of the NLR gas in terms of two easily observed optical emissionline ratios.

\section{CALCULATIONS}

The grid of models was constructed using the code CLOUDY (Ferland 1996), for three different ionizing continua: two power laws $F_{v} \propto v^{-\alpha}$, for $\alpha=1.0$ and 1.5 (turnon and turnoff energies of $0.1 \mathrm{eV}$ and $100 \mathrm{keV}$, respectively) and the option "Table AGN" in CLOUDY, which uses a typical observed AGN continuum (a combination of power laws; see Mathews \& Ferland 1987). The gas densities were varied over the range $10^{2} \mathrm{~cm}^{-3} \leq N \leq 10^{4} \mathrm{~cm}^{-3}$, since previous works (e.g., Schmitt et al. 1994; Storchi-Bergmann et al. 1992; Tsvetanov \& Walsh 1992) indicate that most observed NLR gas densities are in this range. The ionization parameter was varied over the interval $-4.0 \leq$ $\log U \leq-2.0$, which allows the reproduction of the observed range in emission-line ratios for typical NLRs.

As pointed out in $\S 1$, the oxygen abundances obtained in previous works for the $\mathrm{H}$ II regions close to the nuclei of Seyfert galaxies range from about solar to a few times solar, and we have assumed that the NLRs have similar abundance values. The oxygen abundance was thus varied in the range $8.4 \leq 12+\log (\mathrm{O} / \mathrm{H}) \leq 9.4$. The adopted solar abundance values are $12+\log (\mathrm{O} / \mathrm{H})=8.91$ and $12+$ $\log (\mathrm{N} / \mathrm{H})=7.98$, from Aller (1987) and Grevesse (1984). All elements, except nitrogen, are assumed to have abundances equal to that of oxygen, relative to the solar value.

Nitrogen was assumed to behave as a secondary element. The main observational constraint on the origin of nitrogen is the behavior of the abundance ratio $\mathrm{N} / \mathrm{O}$ as a function of the overall metallicity, as measured by the $\mathrm{O} / \mathrm{H}$ ratio. In the "simple chemical evolution model" (Edmunds 1990), N/O will be a constant for a primary origin of nitrogen, while $\mathrm{N} / \mathrm{O}$ will be proportional to $\mathrm{O} / \mathrm{H}$ for a secondary origin. Vila-Costas \& Edmunds (1993) have used H II region data for a number of spiral and irregular galaxies to reach the conclusion that the $\mathrm{N} / \mathrm{O}$ and $\mathrm{O} / \mathrm{H}$ ratios can be consistently explained by models in which nitrogen has mostly a secondary origin at high abundances, namely, for $12+$ $\log (\mathrm{O} / \mathrm{H})>8.5$. We have reached a similar conclusion by analyzing the data of a number of nuclear starbursts (Storchi-Bergmann, Calzetti, \& Kinney 1994) and of H II regions in the vicinity of active nuclei (Storchi-Bergmann et al. 1996a, 1996b). As we intend to obtain a calibration for the NLR, which is located in the nuclear region of luminous and metal-rich galaxies, we will thus assume that nitrogen has a secondary behavior also in the NLR, with abundance given by the relation

$$
\log (\mathrm{N} / \mathrm{O})=0.96[12+\log (\mathrm{O} / \mathrm{H})]-9.29,
$$

which was obtained by Storchi-Bergmann et al. (1994) for nuclear starbursts.

Another aspect that has also become evident in recent studies (e.g., Schmitt et al. 1994; Storchi-Bergmann et al. 1992; Mulchaey, Wilson, \& Tsvetanov 1996) is that the nuclear region of Seyfert 2 galaxies is obscured, denoting the presence of dust in the NLR. The presence of dust was considered using the command "grains" in CLOUDY, and depleting the heavy elements' abundances as follows: for a solar-abundance nebula, the abundance of the depleted gas, relative to the solar value is $\mathrm{He}=1.0, \mathrm{C}=0.39, \mathrm{~N}=0.81$, $\mathrm{O}=0.62, \quad \mathrm{Ne}=1.0, \quad \mathrm{Mg}=0.26, \quad \mathrm{Al}=0.01, \quad \mathrm{Si}=0.045$, $\mathrm{S}=0.59, \quad \mathrm{Ar}=0.44, \quad \mathrm{Ca}=0.00009, \quad \mathrm{Fe}=0.0089$, $\mathrm{Na}=0.024$, and $\mathrm{Ni}=0.01$. These values correspond to the observed abundance of the interstellar medium (Cowie \& Songaila 1986). For chemical compositions different from solar, the depletions were scaled accordingly.

\section{DISCUSSION}

Under the above assumptions, the parameters that determine the emission-line ratios of the NLR are the spectrum of the ionizing continuum, the ionization parameter, the chemical abundance, and the gas density.

The most easily observed optical emission lines from the NLR of Seyferts are [O II] $\lambda 3727$ (hereafter [O II]); $\mathrm{H} \beta$; [O III] $\lambda \lambda 4959,5007$ (hereafter [O III]); $\mathrm{H} \alpha ;[\mathrm{N}$ II] $\lambda \lambda 6548$, 6584 (hereafter [N II]); and [S II] $\lambda \lambda 6717,6731$ (hereafter [S II]). From these, a number of line ratios can be obtained, for example, [O $\mathrm{II}] /[\mathrm{O} \mathrm{III}]$, which is independent of the chemical abundance and is a good indicator of the ionization parameter; $[\mathrm{O} \mathrm{III}] / \mathrm{H} \beta$, which depends on all the parameters but is also frequently used as an indicator of the ionization parameter; $[\mathrm{N} \mathrm{II}] / \mathrm{H} \alpha$, mostly sensitive to the gaseous abundance; and [S II] $\lambda 6717 / \lambda 6731$, which is sensitive to the density.

If we adopt the simplifying assumption that the density is indicated by the $[\mathrm{S} \mathrm{II}]$ ratio, and that the ionizing spectrum is also known, then we can choose two other emission-line ratios as indicators of the ionization parameter and chemical abundance. From the theoretical point of view, the ratio $[\mathrm{O} \mathrm{II}] /[\mathrm{O} \mathrm{III}]$ is the best choice for the derivation of the ionization parameter, as it does not depend on the chemical abundance of the gas. But, from the observational point of view, it has the disadvantage of being sensitive to the reddening. In order to avoid this problem, the ratio [O III]/H $\beta$ can be used instead, but it will also be sensitive to the abundance and should be used together with the ratio mostly sensitive to the abundance, which is $[\mathrm{N} \mathrm{II}] / \mathrm{H} \alpha$. These last two ratios have the advantage of not being sensitive to reddening.

In order to derive a chemical abundance calibration for the NLR, we have explored two diagrams: $[\mathrm{N} \mathrm{II]} / \mathrm{H} \alpha$ versus $[\mathrm{O} \mathrm{III}] / \mathrm{H} \beta$ and $\log ([\mathrm{O} \mathrm{II}] /[\mathrm{O} \mathrm{III}])$ versus $\log ([\mathrm{N} \mathrm{II}] / \mathrm{H} \alpha)$. In Figure 1 , we present a grid of models in the $[\mathrm{N} \mathrm{II}] / \mathrm{H} \alpha$ versus $[\mathrm{O} \mathrm{III}] / \mathrm{H} \beta$ plane, for a range of oxygen abundances $8.4 \leq 12+\log (\mathrm{O} / \mathrm{H}) \leq 9.2$. [Hereafter, we will refer to $12+\log (\mathrm{O} / \mathrm{H})$ as $(\mathrm{O} / \mathrm{H})$.] In these models, the gas density is $300 \mathrm{~cm}^{-3}$ (typical for the NLR) and the ionizing continuum is the segmented power law of Mathews \& Ferland (1987) (typical of AGNs). In Figure 2, the same grid of models is plotted in the $\log ([\mathrm{O} \mathrm{II}] /[\mathrm{O} \mathrm{III}])$ versus $\log ([\mathrm{N} \mathrm{II}] / \mathrm{H} \alpha)$ plane, for a range of oxygen abundances $8.4 \leq(\mathrm{O} / \mathrm{H}) \leq 9.4$.

It can be concluded from Figures 1 and 2 that the pair of ratios $[\mathrm{O} \mathrm{III}] / \mathrm{H} \beta,[\mathrm{N}$ II] $] / \mathrm{H} \alpha$ or $\log \left(\left[\begin{array}{ll}\mathrm{O} & \mathrm{II}\end{array}\right] /[\mathrm{O} \mathrm{III}]\right), \log$ $([\mathrm{N}$ III $] / \mathrm{H} \alpha)$ can determine an abundance value. Note the clean sequencing of models in terms of the abundance values in the figures, specially in the latter diagram (Fig. 2). This diagram is particularly recommended for high abundances $[(\mathrm{O} / \mathrm{H})>9.2]$, because of some degeneracy of the models in the first diagram (Fig. 1). 




FIG. 1.-Sequence of photoionization models in which the gas density is $300 \mathrm{~cm}^{-3}$, the dust-to-gas ratio is solar, and the ionization parameter is varied in the range $-4.0 \leq \log U \leq-2.0$. Each sequence corresponds to a different chemical abundance, from $12+\log (\mathrm{O} / \mathrm{H})=8.4$ (bottom) to 9.2 (top).

We next fitted two-dimensional second-order polynomials to each of the diagrams in order to derive a calibration for the oxygen abundance in terms of the above emission-line ratios. For the first diagram (Fig. 1) we obtain, for $x \equiv[\mathrm{N} \mathrm{III}] / \mathrm{H} \alpha$ and $y \equiv[\mathrm{O} \mathrm{III}] / \mathrm{H} \beta$, interpolating in the interval $8.4 \leq(\mathrm{O} / \mathrm{H}) \leq 9.2$,

$$
\begin{aligned}
(\mathrm{O} / \mathrm{H})= & 8.34+0.212 x-0.012 x^{2}-0.002 y \\
& +0.007 x y-0.002 x^{2} y+6.52 \times 10^{-4} y^{2} \\
& +2.27 \times 10^{-4} x y^{2}+8.87 \times 10^{-5} x^{2} y^{2} .
\end{aligned}
$$

For the second diagram, the best fit obtained for $u \equiv$ $\log ([\mathrm{O} \mathrm{II}] /[\mathrm{O} \mathrm{III}])$ and $v \equiv \log ([\mathrm{N} \mathrm{II}] / \mathrm{H} \alpha)$ in the interval

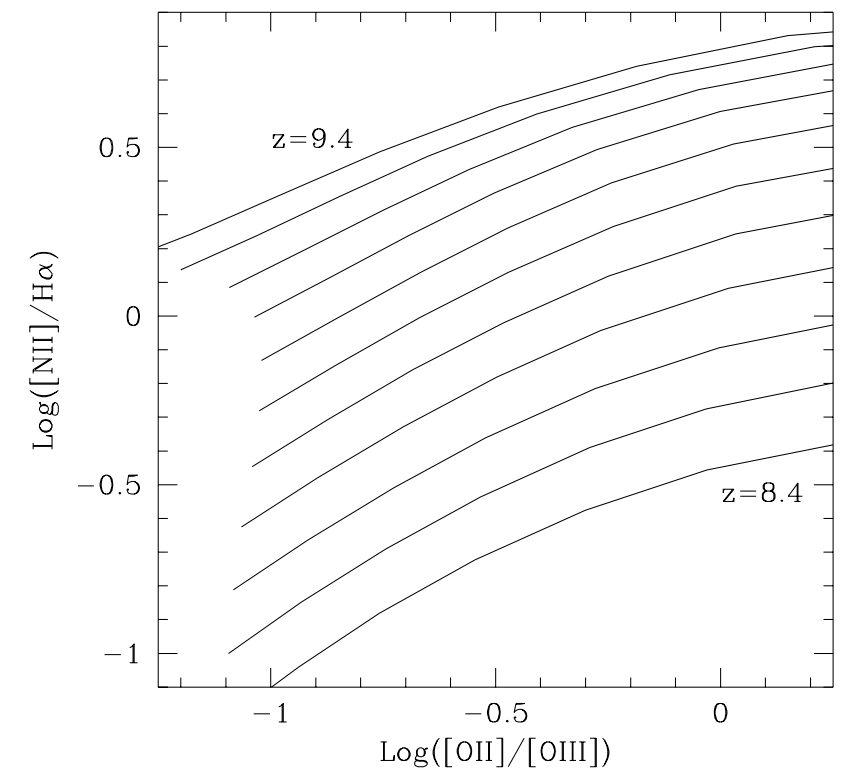

Fig. 2.-Same as Fig. 1, for the $\log ([\mathrm{O} \mathrm{II}] /[\mathrm{O} \mathrm{III}])$ vs. $\log ([\mathrm{N} \mathrm{II}] / \mathrm{H} \alpha)$ plane, in the range $8.4 \leq 12+\log (\mathrm{O} / \mathrm{H}) \leq 9.4$.

$$
\begin{aligned}
& 8.4 \leq(\mathrm{O} / \mathrm{H}) \leq 9.4 \text { is } \\
& \begin{aligned}
(\mathrm{O} / \mathrm{H}) & =8.643-0.275 u+0.164 u^{2}+0.655 v-0.154 u v \\
& -0.021 u^{2} v+0.288 v^{2}+0.162 u v^{2}+0.0353 u^{2} v^{2} .
\end{aligned}
\end{aligned}
$$

For both expressions, the fitted values are within 0.05 dex of the model values, with $\chi^{2}$ of $1.04 \times 10^{-3}$ and $6.9 \times 10^{-4}$, respectively.

The dependence of the calibrations on the density can be quantified as long as the density stays approximately within the range $100 \mathrm{~cm}^{-3} \leq N \leq 10,000 \mathrm{~cm}^{-3}$. For larger densities, $[\mathrm{N} \mathrm{II}] / \mathrm{H} \alpha$ is not a good abundance indicator anymore, because it is suppressed as its critical density is $8.6 \times 10^{4} \mathrm{~cm}^{-3}$, and the calibrations are not valid. As the density is increased, both $[\mathrm{O} \mathrm{III}] / \mathrm{H} \beta$ and $[\mathrm{N} \mathrm{II}] / \mathrm{H} \alpha$ ratios increase systematically, and the dependence of the two calibrations on the density is approximately linear in the logarithm of the density. This dependence can be incorporated into our calibrations, as

$$
(\mathrm{O} / \mathrm{H})_{\mathrm{final}}=(\mathrm{O} / \mathrm{H})-0.1 \log (N / 300),
$$

where $N$ is the gas density in $\mathrm{cm}^{-3}$ and the equation is valid for $100 \mathrm{~cm}^{-3} \leq N \leq 10,000 \mathrm{~cm}^{-3}$.

Calibrations using power-law ionizing continua $F_{v} \propto$ $v^{-\alpha}$, for $\alpha=1$ and 1.5, were also obtained. As compared with the above calibrations, for which we used the typical AGN continuum of Mathews \& Ferland (1987), it can be concluded that they yield systematically larger values for the calculated abundances. The second calibration [in terms of $\log ([\mathrm{N} \mathrm{II}] / \mathrm{H} \alpha)$ and $\log ([\mathrm{O} \mathrm{II}] /[\mathrm{O}$ III $])]$ is less dependent on the ionizing continuum, yielding abundance values from 0.1 to 0.3 dex larger with the power law than with the AGN continuum. For the first calibration, the difference is increased, reaching values larger than 0.5 dex. We point out that, although the shape of the ionizing continuum in AGNs is not very well established, UV and X-ray observations of at least the brightest AGNs show that it is closer to a composition of power laws than to a unique power law (see, e.g., Sanders et al. 1989; Alloin et al. 1995), favoring the "AGN continuum" used above over the single power laws.

\section{COMPARISON WITH OBSERVATIONS}

In order to verify the validity of the above calibrations, we have collected from the literature the emission-line ratios of the NLR of a few Seyfert galaxies and LINERs for which there are determinations of the oxygen and nitrogen abundances for $\mathrm{H}$ II regions close to the NLR. In this way, the NLR abundances could be extrapolated from those of the $\mathrm{H}$ iI regions.

The collected data are shown in Table 1, along with the Hubble type and absolute magnitude for each galaxy. The emission-line fluxes are listed relative to $\mathrm{H} \beta$ and have been corrected for reddening, and their source is listed in the last column of the table. Also listed are the electron densities calculated from the [S II] $\lambda \lambda 6716 / 6731$ line ratios, as in Figure 5.3 of Osterbrock (1989). In applying the correction to the $(\mathrm{O} / \mathrm{H})$ due to the density dependence (eq. [4]), we will assume that the gas density is approximately the same as the electron density $\left(N \approx N_{e}\right)$.

Typical radial abundance gradients for normal galaxies range between 0.05 and 0.1 dex kpc ${ }^{-1}$ (see, e.g., Vila-Costas \& Edmunds 1992; Kennicutt et al. 1993). Schmitt et al. (1994) and Storchi-Bergmann et al. (1996a, 1996b) have 
TABLE 1

EMission-Line FluXES Relative to H $\beta$ (REDDENING-FREE)

\begin{tabular}{|c|c|c|c|c|c|c|c|c|}
\hline Galaxy & Type $^{\mathrm{a}}$ & $M_{B}^{\mathrm{a}, \mathrm{b}}$ & {$[\mathrm{O}$ II $]$} & {$[\mathrm{O} \mathrm{III}]$} & $\mathrm{H} \alpha$ & {$[\mathrm{N}$ II $]$} & $\begin{array}{c}N_{e} \\
\left(\mathrm{~cm}^{-3}\right)\end{array}$ & Reference \\
\hline \multicolumn{9}{|l|}{ Seyfert galaxies: } \\
\hline IC $1816 \ldots$. & $\mathrm{Sab}$ & -20.3 & $2.0 \pm 0.2$ & $17.9 \pm 0.5$ & $2.9 \pm 0.2$ & $6.9 \pm 0.3$ & 650 & 1 \\
\hline NGC $1068 \ldots \ldots$ & (R)SA(rs)b & -21.3 & $1.2 \pm 0.2$ & $16.5 \pm 0.4$ & $2.6 \pm 0.2$ & $6.1 \pm 0.3$ & 8000 & 2 \\
\hline NGC $1386 \ldots \ldots$ & $\mathrm{Sa}$ & -19.0 & $2.2 \pm 0.2$ & $15.9 \pm 0.4$ & $3.0 \pm 0.2$ & $5.0 \pm 0.3$ & 650 & 1 \\
\hline NGC $1566 \ldots \ldots$ & $\mathrm{SAB}(\mathrm{rs}) \mathrm{bc}$ & -21.2 & $2.0 \pm 0.3$ & $15.0 \pm 0.4$ & $3.1 \pm 0.4$ & $4.1 \pm 0.4$ & 650 & 3 \\
\hline NGC $3081 \ldots \ldots$ & S0/a & -20.1 & $1.5 \pm 0.2$ & $15.1 \pm 0.4$ & $3.0 \pm 0.2$ & $3.7 \pm 0.2$ & 200 & 1 \\
\hline NGC $5643 \ldots \ldots$ & $\mathrm{SAB}(\mathrm{rs}) \mathrm{c}$ & -20.3 & $5.20 \pm 0.5$ & $16.4 \pm 0.4$ & $3.0 \pm 0.2$ & $4.2 \pm 0.2$ & 200 & 4 \\
\hline NGC $6814 \ldots \ldots$ & $\mathrm{SAB}(\mathrm{rs}) \mathrm{bc}$ & -19.5 & $3.3 \pm 0.2$ & $15.8 \pm 0.4$ & $2.8 \pm 0.2$ & $6.0 \pm 0.3$ & 650 & 5 \\
\hline \multicolumn{9}{|l|}{ LINERs: } \\
\hline NGC $1097 \ldots \ldots$ & $\mathrm{SB}(\mathrm{rs}) \mathrm{bc}$ & -21.0 & $4.0 \pm 0.4$ & $5.1 \pm 0.3$ & $2.9 \pm 0.2$ & $7.2 \pm 0.4$ & 400 & 6 \\
\hline NGC $1326 \ldots \ldots$ & RSBa & -19.7 & $13.2 \pm 0.6$ & $2.6 \pm 0.2$ & $2.9 \pm 0.2$ & $5.8 \pm 0.3$ & 650 & 1 \\
\hline NGC $1598 \ldots \ldots$ & $\mathrm{Sbc}$ & -20.2 & $0.7 \pm 0.1$ & $2.3 \pm 0.2$ & $3.0 \pm 0.2$ & $4.7 \pm 0.2$ & 100 & 1 \\
\hline NGC $1672 \ldots \ldots$ & $\mathrm{SB}(\mathrm{s}) \mathrm{b}$ & -19.9 & $1.3 \pm 0.1$ & $2.1 \pm 0.2$ & $2.9 \pm 0.2$ & $4.1 \pm 0.2$ & 250 & 6 \\
\hline
\end{tabular}

${ }^{a}$ From Storchi-Bergmann et al. 1996a, 1996b, except for NGC 1068, 1566, 5643, and 6814, for which we have used the NASA/IPAC Extragalactic Database.

${ }^{b} H_{0}=75 \mathrm{~km} \mathrm{~s}^{-1} \mathrm{Mpc}^{-1}$.

REFERENCES.- (1) Storchi-Bergmann et al. 1996a; (2) Koski 1978; (3) Alloin et al. 1985; (4) Schmitt et al. 1994; (5) our unpublished data;(6) Storchi-Bergmann et al. 1996b.

shown that, for the Seyfert 2 NGC 5643 and the LINERs NGC 1097, NGC 1672, and NGC 1598, the observed gradients are similar to those typical of normal galaxies with the same morphological type. Here we thus adopt the latter hypothesis in the extrapolation of the NLR abundances, for the cases in which a gradient is not available.

In Table 2, we present the resulting $(\mathrm{O} / \mathrm{H})$ values of the NLRs obtained with each of the proposed calibrationsequations (2), (3), and (4) above, together with the derived values from the extrapolation using the abundances of the $\mathrm{H}$ II regions. We also show, in the last two columns of the table, the adopted gradient in $(\mathrm{O} / \mathrm{H})$ and the references from which the H II regions' abundances and gradients were obtained. Errors in the calibrations have been calculated from the errors in the emission-line ratios, while errors in the extrapolated values have been estimated from the errors in the $\mathrm{H}$ II regions' abundances and gradients.

The $(\mathrm{O} / \mathrm{H})$ values obtained from the two calibrations are plotted against those extrapolated from the $\mathrm{H}$ iI regions in Figure 3. It can be seen that, within the errors, there is a reasonable agreement between the extrapolated and calculated values for the Seyfert 2 galaxies, with maximum differences of $\sim 0.2$ dex between the calculated and extrapolated values, indicating that the calibration works very well for the Seyfert 2 galaxies.

The $(\mathrm{O} / \mathrm{H})$ values obtained using the calibrations can also be compared with previous determinations via detailed modeling of the NLR, which we have for NGC 5643 (Schmitt et al. 1994) and another Seyfert 2 galaxy, NGC 3281 (Storchi-Bergmann et al. 1992). In both cases, the models indicated solar abundance for the gas $(\mathrm{O} / \mathrm{H})=8.91$, while the calibrations yield $(\mathrm{O} / \mathrm{H})=9.00$ (eqs. [2] and [4]) and $(\mathrm{O} / \mathrm{H})=8.94$ (eqs. [3] and [4]) for NGC 5643 and $(\mathrm{O} / \mathrm{H})=8.81$ and 9.03 , respectively, for NGC 3281, which confirm the validity of the calibrations.

On the other hand, for all four LINERs the calibrations yield values that are systematically lower than those of the Seyfert galaxies. But we note that Storchi-Bergmann et al. (1996a, 1996b) have concluded that the LINER nucleus of NGC 1672 presents emission-line ratios that can be repro-

TABLE 2

OXYGEN ABUNDANCES OF THE NLR

\begin{tabular}{|c|c|c|c|c|c|}
\hline Galaxy & $\mathrm{O} / \mathrm{H}^{\mathrm{a}}$ & $\mathrm{O} / \mathrm{H}^{\mathrm{b}}$ & $\mathrm{O} / \mathrm{H}^{\mathrm{c}}$ & $\Delta(\mathrm{O} / \mathrm{H})^{\mathrm{d}}$ & References \\
\hline \multicolumn{6}{|l|}{ Seyfert galaxies: } \\
\hline IC 1816. & $9.35 \pm 0.09$ & $9.34 \pm 0.04$ & $9.3 \pm 0.1$ & -0.06 & 1 \\
\hline NGC $1068 \ldots \ldots$ & $9.14 \pm 0.08$ & $9.35 \pm 0.06$ & $9.1 \pm 0.1$ & & 2,3 \\
\hline NGC $1386 \ldots \ldots$ & $9.02 \pm 0.06$ & $9.15 \pm 0.04$ & $8.9 \pm 0.1$ & -0.06 & 1 \\
\hline NGC $1566 \ldots \ldots$ & $8.87 \pm 0.07$ & $9.07 \pm 0.07$ & $9.1 \pm 0.2$ & -0.08 & 4 \\
\hline NGC $3081 \ldots \ldots$ & $8.90 \pm 0.05$ & $9.17 \pm 0.05$ & $9.1 \pm 0.2$ & -0.02 & 1,5 \\
\hline NGC $5643 \ldots \ldots$ & $9.00 \pm 0.05$ & $8.95 \pm 0.04$ & $9.0 \pm 0.1$ & -0.05 & 6 \\
\hline NGC $6814 \ldots \ldots$ & $9.15 \pm 0.07$ & $9.14 \pm 0.05$ & $9.3 \pm 0.2$ & -0.07 & 2,5 \\
\hline \multicolumn{6}{|l|}{ LINERs: } \\
\hline NGC $1097 \ldots \ldots$ & $8.84 \pm 0.04$ & $8.97 \pm 0.04$ & $9.3 \pm 0.1$ & -0.05 & 7 \\
\hline NGC $1326 \ldots \ldots$ & $8.70 \pm 0.03$ & $8.70 \pm 0.03$ & $9.0 \pm 0.1$ & -0.06 & 1 \\
\hline NGC $1598 \ldots \ldots$ & $8.71 \pm 0.02$ & $9.03 \pm 0.04$ & $9.2 \pm 0.1$ & -0.06 & 1 \\
\hline NGC $1672 \ldots \ldots$ & $8.64 \pm 0.02$ & $8.83 \pm 0.03$ & $9.2 \pm 0.2$ & -0.06 & 7 \\
\hline
\end{tabular}

a The value of $12+\log (\mathrm{O} / \mathrm{H})$ from the first calibration (eqs. [2] and [4]).

b The value of $12+\log (\mathrm{O} / \mathrm{H})$ from the second calibration (eqs. [3] and [4]).

${ }^{c}$ Extrapolating from the $\mathrm{H}$ in regions.

d Adopted gradient in dex $\mathrm{kpc}^{-1}$.

REFERENCES.- (1) Storchi-Bergmann et al. 1996a; (2) Evans \& Dopita 1987; (3) Oey \& Kennicutt 1993; (4) Hawley \& Phillips 1980; (5) Vila-Costas \& Edmunds 1992; (6) Schmitt et al. 1994; (7) Storchi-Bergmann et al. 1996b. 


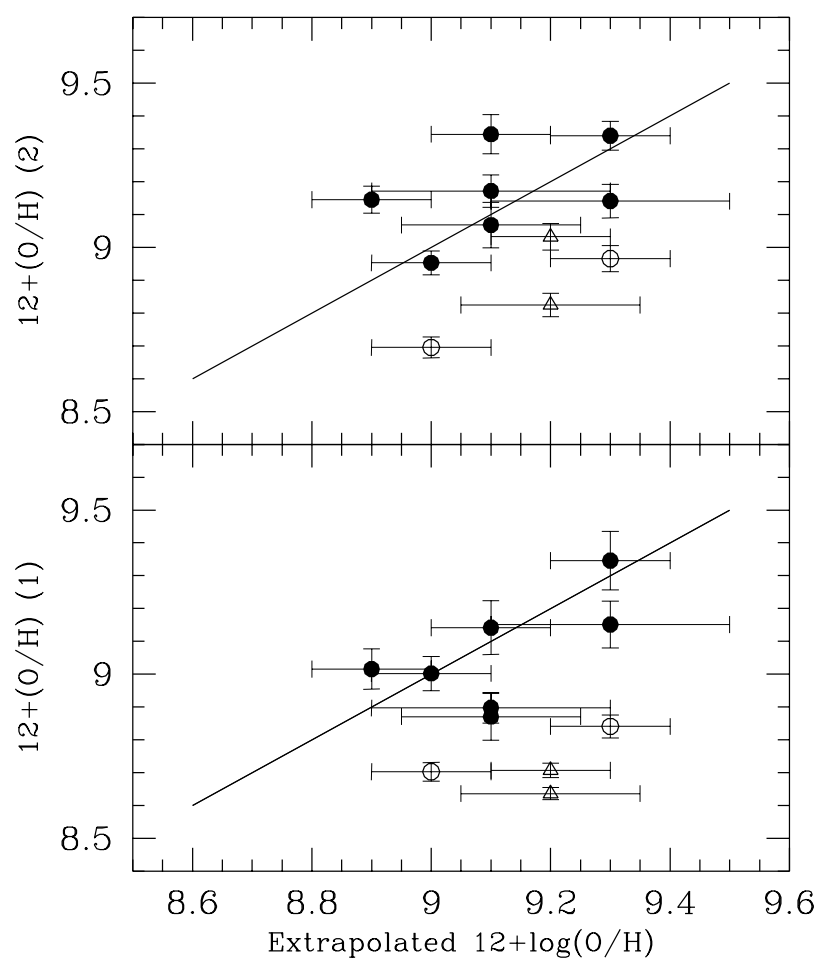

FIG. 3.-Oxygen abundance values $12+\log (\mathrm{O} / \mathrm{H})$ for the NLR, obtained from the two proposed calibrations, plotted against the values obtained from the $\mathrm{H}$ II regions. Filled symbols represent the Seyfert 2 galaxies, and open symbols the LINERs. The triangles represent the LINERs for which there are signatures of recent star formation in the nucleus. Bottom, first calibration, involving the line ratios $[\mathrm{N} \mathrm{III}] / \mathrm{H} \alpha$ and $[\mathrm{O} \mathrm{III}] / \mathrm{H} \beta$ (Fig. 1); top, second calibration, involving $\log ([\mathrm{N} \mathrm{II}] / \mathrm{H} \alpha)$ and $\log ([\mathrm{O}$ II $] /[\mathrm{O}$ III]) (Fig. 2). Both calibrations have been corrected by a small dependence on the gas density (see text). The loci of equal values for the two quantities are drawn as a line for comparison.

duced with photoionization by hot stars, and the absorption spectra from both this nucleus and that of NGC 1598 present signatures of young stars. From the discussion above, the calibrations would not be valid for these two cases, identified by triangles in Figure 3. But they should be valid for the other two LINER nuclei. As there is no systematic difference between the extrapolated nuclear abundances for the NLRs of Seyfert galaxies and LINERs, the different behavior of the LINERs in the diagrams suggests a difference between the physical conditions and/or ionizing source in these objects, as compared with the Seyfert galaxies. One possibility is that the energy distribution responsible for the photoionization of the gas in LINERs is an absorbed continuum. Matter-bounded clouds from the BLR (Ferland et al. 1996) or from the NLR (Binette et al. 1996) would be responsible for the absorption. Nevertheless, our results are based on observations of only two objects. More LINERs need to be subjected to this kind of study before a firm conclusion can be reached.

We also show, in Figure 4, the results of the two calibrations plotted against each other. Although the correlation is not perfect (as the calibrations are fits to the sequences of models), the Spearman correlation coefficient between the two calibrations is $r_{\mathrm{S}}=0.80$, indicating a good correlation. It can also be noted that there is a systematic shift between the two calibrations, such that the second gives $(\mathrm{O} / \mathrm{H})$ values on average 0.11 dex larger than the first calibration. As judged from the comparison with the

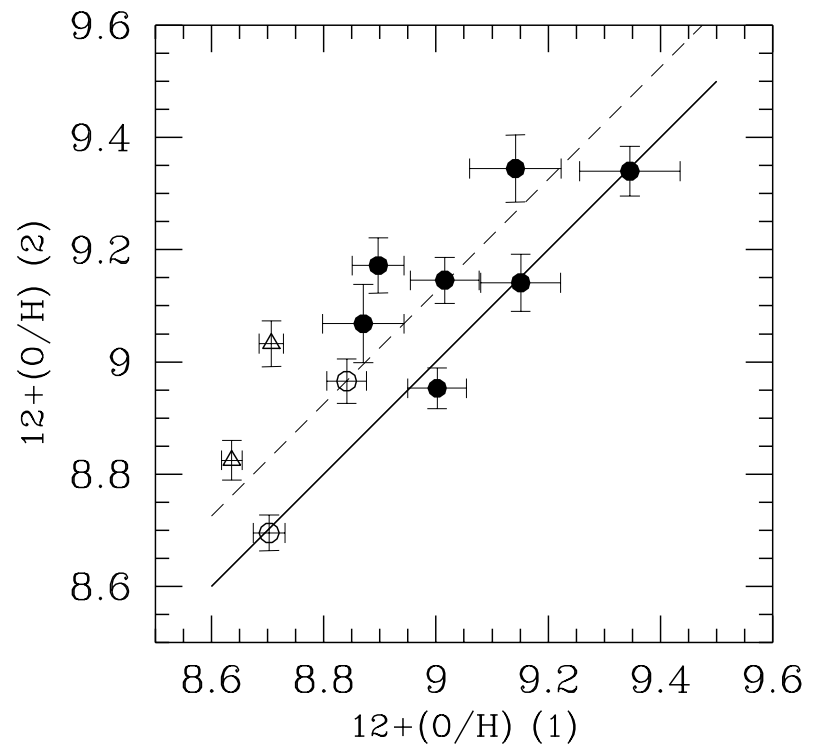

FIG. 4.-Oxygen abundance values $12+\log (\mathrm{O} / \mathrm{H})$, obtained with the second calibration (Fig. 2), plotted against the values obtained with the first calibration (Fig. 1). For comparison, the loci of equal values for the two quantities are drawn as a solid line, and those corresponding to a systematic shift of the second calibration to $12+\log (\mathrm{O} / \mathrm{H})$ values $0.11 \mathrm{dex}$ larger are drawn as a dashed line. Symbols are as in Fig. 3.

observations, the $(\mathrm{O} / \mathrm{H})$ value to be adopted when both calibrations can be used is the average of the two.

As pointed out in the previous section, we have also calculated calibrations for power-law ionizing continua instead of the "AGN continuum" used to obtain the calibrations of equations (2) and (3). Nevertheless, comparison of the calculated $(\mathrm{O} / \mathrm{H})$ with the observed ones (from the $\mathrm{H}$ II regions) gives a much poorer agreement. The calibration in terms of $\log ([\mathrm{N} \mathrm{II}] / \mathrm{H} \alpha)$ and $\log ([\mathrm{O} \mathrm{II}] /[\mathrm{O} \mathrm{III}])$ (second calibration) gives a somewhat better result than the one in terms of $[\mathrm{N} \mathrm{II}] / \mathrm{H} \alpha$ and $[\mathrm{O} \mathrm{III}] / \mathrm{H} \beta$ (first calibration): although the calculated $(\mathrm{O} / \mathrm{H})$ are systematically overestimated when compared with the observed ones, the difference stays within the range 0.1 to 0.3 dex. But the first calibration gives $(\mathrm{O} / \mathrm{H})$ values larger than the observed ones by $0.5 \mathrm{dex}$ in some cases. These results suggest that the "AGN continuum" is a better representation of the ionizing continuum than the single power laws for the sample galaxies.

It is important to point out that there may be at least one source of confusion in observed emission-line ratios from the NLR: the proximity of $\mathrm{H}$ II regions, such that the observed emission-line ratios could be the result of a mixture of gas photoionized by an AGN continuum and by blue stars. In principle, such cases could be sorted out using diagnostic diagrams (Baldwin, Phillips, \& Terlevich 1981; Veilleux \& Osterbrock 1987). The calibrations are valid only for those cases in which the emission-line ratios are clearly located in the region of the diagrams corresponding to the Seyfert galaxies. In the "mixed" cases, the line ratios are intermediate between those of Seyfert galaxies and those of $\mathrm{H}$ II regions, and the calibrations are not valid.

\section{SUMMARY AND FINAL REMARKS}

We have collected chemical abundance data of $\mathrm{H}$ II regions in the vicinity of the nuclei for a sample of 11 active galaxies and have used them to extrapolate the chemical 
abundances of the corresponding NLRs. These abundances were used to test calibrations that allow the determination of the chemical abundance of the NLR of Seyfert galaxies in terms of two easily observed optical emission-line ratios. Two calibrations were obtained, the first involving a linear combination of the ratios $[\mathrm{N} \mathrm{II}] / \mathrm{H} \alpha$ and $[\mathrm{O} \mathrm{III]} / \mathrm{H} \beta$, and the second a linear combination of the decimal logarithms of $[\mathrm{N}$ II $] / \mathrm{H} \alpha$ and $[\mathrm{O}$ II $] /[\mathrm{O}$ III $]$. Although the first calibration involves ratios less sensitive to the reddening, we have concluded that the second calibration is less sensitive to the ionizing continuum spectrum. Thus, whenever possible, both calibrations should be used (eqs. [2], [3], and [4]) and the $(\mathrm{O} / \mathrm{H})$ abundance calculated as the average between the two values. The calibrations work well for all of the Seyfert galaxies, suggesting that the hypotheses in the modeling of the NLR are valid for most Seyfert galaxies.

The calibrations seem not to work for the LINERs, suggesting that different ionization mechanisms occur in these objects. Nevertheless, more LINERs need to be studied before a firm conclusion can be reached, as there are only four LINERs in the sample, and for two of them there seems to be contamination of the NLR by surrouding $\mathrm{H}$ II regions, and the calibrations do not work in these cases.

It would be important to test the calibrations in more objects, with a larger abundance range, but presently there are no other active galaxies for which there are determi- nations of the chemical abundance of their $\mathrm{H}$ II regions in the literature. A first step to look more closely into the abundances of $\mathrm{H}$ II regions in a larger number of Seyfert galaxies has been made by Evans et al. (1996), who have recently published an atlas with the optical positions of $\mathrm{H}$ II regions in 17 Seyfert galaxies. The following step would be to make spectroscopic observations of the individual $\mathrm{H}$ II regions.

In summary, we point out that determining the chemical abundance of the NLR in Seyfert galaxies is not an easy task. In previous works we have explored two methods: modeling of individual NLRs and abundance determination of $\mathrm{H}$ II regions in the vicinity of the NLR. In this work we propose an alternative method: simple calibrations to be used when just a quick estimate of the chemical abundance is necessary.

This research received partial support from the Brazilian institutions CNPq, Finep, and FAPERGS. We acknowledge fruitful discussions with $\mathrm{L}$. Binette, and the suggestions of an anonymous referee, which helped to improve the paper. This research has made use of the NASA/IPAC Extragalactic Database (NED), which is operated by the Jet Propulsion Laboratory, California Institute of Technology, under contract with the National Aeronautics and Space Administration.

\section{REFERENCES}

Aller, L. H. 1987, in Spectroscopy of Astrophysical Plasmas, ed. A. Dalgarno \& D. Layzer (Cambridge: Cambridge Univ. Press), chap. 4

Alloin, D., Pelat, D., Phillips, M., \& Whittle, M. 1985, ApJ, 288, 205

Alloin, D., et al. 1995, A\&A, 293, 293

Baldwin, J. A., Phillips, M. M., \& Terlevich, R. 1981, PASP, 93, 5

Binette, L., Robinson, A., \& Courvoisier, T. J.-L. 1988, A\&A, 194, 65

Binette, L., Wilson, A. S., \& Storchi-Bergmann, T. 1996, A\&A, 312, 365

Cowie, L. L., \& Songaila, A. 1986, ARA\&A, 24, 499

Dopita, M. A., \& Sutherland, R. S. 1995, ApJ, 455, 468

Edmunds, M. G. 1990, MNRAS, 246, 678

Evans, I. N., \& Dopita, M. 1987, ApJ, 319, 662

Evans, I. N., Koratkar, A. P., Storchi-Bergmann, T., Kirkpatrick, H., Heckman, T. M., \& Wilson, A. S. 1996, ApJS, 105, 93

Ferland, G. J. 1996, HAZY, a Brief Introduction to Cloudy (Univ. Kentucky Phys. Astron. Dept. Internal Rep.)

Ferland, G. J., Baldwin, J. A., Korista, K. T., Hamann, F., Carswell, R. F.,

Phillips, M., Wilkes, B., \& Williams, R. E. 1996, ApJ, 461, 683

Ferland, G. J., \& Netzer, H. 1983, ApJ, 264, 105

Ferland, G. J., \& Osterbrock, D. E. 1986, ApJ, 300, 658

Grevesse, N. 1984, in Frontiers of Astronomy and Astrophysics, ed.

R. Pallavicini (Florence: Soc. Astron. Italiana), 71

Halpern, J. P., \& Steiner, J. 1983, ApJ, 269, L37

Hawley, S. A., \& Phillips, M. M. 1980, ApJ, 235, 783

Kennicutt, R. C., Oey, M. S., Zaritsky, D., \& Huchra, J. P. 1993, Rev.

Mexicana Astron. Astrofis., 27, 21

Koski, A. 1978, ApJ, 223, 56

Mathews, W. G., \& Ferland, G. J. 1987, ApJ, 323, 456
Mulchaey, J. S., Wilson, A. S., \& Tsvetanov, Z. 1996, ApJ, 467, 197

Oey, M. S., \& Kennicutt, R. C., Jr. 1993, ApJ, 411, 137

Osterbrock, D. E. 1989, Astrophysics of Gaseous Nebulae and Active Galactic Nuclei (Mill Valley, CA: Univ. Sci.)

Sanders, D. B., Phinney, E. S., Neugebauer, G., Soifer, B. T., \& Matthews, K. 1989, ApJ, 347, 29

Schmitt, H. R., Storchi-Bergmann, T., \& Baldwin, J. A. 1994, ApJ, 423, 237

Stasińska, G. 1984, A\&A, 135, 341

Storchi-Bergmann, T., Ardila, A. R., Schmitt, H. R., Wilson, A. S., \& Baldwin, J. A. 1996a, ApJ, 472, 83

Storchi-Bergmann, T., Calzetti, D., \& Kinney, A. L. 1994, ApJ, 429, 572

Storchi-Bergmann, T., \& Pastoriza, M. G. 1990, MNRAS, 245, 749

Storchi-Bergmann, T., Wilson, A. S., \& Baldwin, J. A. 1992, ApJ, 396, 45 . 1996b, ApJ, 460, 252

Sutherland, R. S., Bicknell, G. V., \& Dopita, M. A. 1993, ApJ, 414, 510

Tsvetanov, Z., \& Walsh, J. R. 1992, ApJ, 386, 485

Veilleux, S., \& Osterbrock, D. E. 1987, ApJS, 63, 295

Viegas, S. M., \& de Gouveia Dal Pino, E. M. 1992, ApJ, 384, 467

Viegas-Aldrovandi, S. M. 1988, ApJ, 330, L9

Viegas-Aldrovandi, S. M., \& Contini, M. 1989, ApJ, 339, 689

Viegas-Aldrovandi, S. M., \& Gruenwald, R. B. 1990, ApJ, 360, 474

Vila-Costas, M. B., \& Edmunds, M. G. 1992, MNRAS, 259, 121

.1993, MNRAS, 265, 199

Wilson, A. S. 1996, Vistas Astron., 40, 63 